\title{
Postherpetic foot drop in an elderly case: undiagnosed diabetes mellitus?
}

\author{
Zeynep Öztürk, ${ }^{1}$ Ayşe Türkylmaz² \\ ${ }^{1}$ Department of Clinical Pharmacology and Toxicology, İzmir Atatürk Training and Research Hospital, İzmir, Turkey \\ ${ }^{2}$ Department of Neurology, Atatürk Research Hospital, İzmir Atatürk Training and Research Hospital, İzmir, Turkey \\ Received: May 2015 Accepted: September 2015
}

\begin{abstract}
Postherpetic neuralgia is the most commonly seen complication of herpes zoster, primarily in elderly and immunocompromised patients. However, motor involvement due to herpes zoster is very rare. A 65-year-old male patient presented with left leg pain, numbness and difficulty walking. He was diagnosed with having herpes zoster and treated with valacyclovir. Despite anti-convulsant and physical therapy, the patient developed foot drop and had an unexplained weight loss. Clinical and laboratory findings suggested previously undiagnosed diabetes mellitus. Three weeks after initiation of insulin treatment, he became normoglycemic, his pain was reduced, and foot drop disappeared almost completely. Postherpetic motor involvement as an unusual complication may signal an underlying systemic disease, particularly in elderly.
\end{abstract}

Keywords: Diabetes; foot drop; herpes zoster; pain; postherpetic paresis.

Herpes zoster is a viral disease characterized by a painful skin rash which can be more severe in older people than children. Postherpetic neuralgia occurs in approximately $20 \%$ of patients and can decrease quality of life, particularly in the elderly. However, zoster paresis is a rare complication of herpes zoster and focal motor weakness does not always present simultaneously with skin lesions. ${ }^{[1]}$ There is a strong correlation between increasing age and herpes zoster. $^{[2,3]}$ Neuropathic pain in herpes zoster can be a symptom, manifestation or complication which is often difficult to treat. Medication alone may not be enough to relieve pain. A combination therapy is often more effective than monotherapy and recommended for chronic pain management. ${ }^{[4]}$

Neuropathic pain is a complex pain associated with nerve injuries, malfunctions, and excitability changes. Effective control of the underlying disease is important in neuropathic pain management. Symptomatic treatment includes antiepileptic drugs, tricyclic antidepressants, serotonin-norepinephrine reuptake inhibitors and physical therapy. Standard analgesics used alone, such as non-steroidal antiinflammatory drugs, are often ineffective for treating neuropathic pain.
Neuropathic pain is caused by primary dysfunction of the peripheral or central nervous system. Diabetes mellitus, infection, cancer, autoimmune disease, hereditary factors, trauma, vitamin deficiencies and neurotoxicity can cause peripheral neuropathic pain. Central neuropathic pain can be secondary to brain infarction, spinal cord injury, multiple sclerosis or other dysfunction of the central nervous system. ${ }^{[5]}$ Symptoms of peripheral neuropathic pain in the elderly are common. However, pain is not a natural result of aging. ${ }^{[6,7]}$

Herein, we report a case of postherpetic paresis and drop foot as an unusual complication in whom late clinical signs suggested previously undiagnosed diabetes mellitus.

\section{CASE REPORT}

A 65-year-old male patient who developed left leg pain and numbness presented with difficulty standing up and walking. He did not respond to non-steroidal anti-inflammatory drugs (NSAIDs) and many vesicles appeared on the same leg two weeks after starting this treatment. There was no other drug exposure or known systemic illness in his past

Corresponding author: Zeynep Öztürk, MD. İzmir Atatürk Ĕgitim ve Araştırma Hastanesi, Klinik Farmakoloji ve Toksikoloji Kliniği, 35360 Basın Sitesi, İzmir, Turkey. e-mail: dr.zeyneb@hotmail.com 
Table 1. Nerve conduction study

\begin{tabular}{|c|c|c|c|}
\hline Nerve & Amplitude $(\mathrm{mV}-\mu \mathrm{V})$ & Latency (msec) & Nerve conduction velocity $(\mathrm{m} / \mathrm{sec})$ \\
\hline Median motor & 11 & 2.7 & 51 \\
\hline Median sensory & 13 & 3.1 & 42 \\
\hline Ulnar motor & 7.5 & 2.2 & 52 \\
\hline Ulnar sensory & 15 & 2.6 & 49 \\
\hline Posterior tibial motor & 5 & 4.1 & 39 \\
\hline Sural sensory & 11 & 3.4 & 47 \\
\hline Peroneal sensory & No response & & \\
\hline Peroneal mixed & No response & & \\
\hline \multicolumn{4}{|l|}{ Peroneal motor } \\
\hline ankle/above the head of fibula/ & $0.4 / 0.4 / 0.3$ & 3.5 (ankle) & 12.9 (above the head of fibula) \\
\hline below the head of fibula & & & 16.0 (below the head of fibula) \\
\hline
\end{tabular}

medical history. The patient was diagnosed as having herpes zoster by a dermatological examination and treated with valacyclovir for seven days ( $3 \mathrm{~g} /$ day oral). Valacyclovir treatment reduced the severity of skin lesions. However, leg pain and motor symptoms such as weakness, numbness and tingling continued to get worse over time. He was referred to the neurology outpatient clinic since he was unable to flex his left foot and had steppage gait. The patient had no complaints such as headache, nausea or vomiting. Physical examination revealed the weakness of left ankle dorsiflexion and eversion. Ankle plantar flexion and foot inversion were normal. In the manual muscle test, muscular strength was reduced to $1 / 5$ (left/right) as compared to the normal side for the anterior tibial, extensor hallucis longus and peroneus longus muscles. There was sensory loss on the anterolateral side of leg and dorsum of the foot. Sensation on the plantar surfaces of the foot and toes was spared. Deep tendon reflexes were normoactive, and knee flexion and hip abduction were normal bilaterally. The nerve conduction velocities (NCVs) of peroneal motor, mixed and sensory nerve were diminished (Table 1). Needle electromyography (EMG) of left tibialis anterior and peroneus longus muscles showed increased insertional activity, fibrillation $(2+)$, positive sharp waves (2+) and reduced recruitment (Table 2). There were no signs of tibialis posterior dysfunction, and no abnormalities were found in the biceps femoris short head muscle. Three weeks after the appearance of the vesicular lesions, the EMG study confirmed the partial axonal degeneration of the left peroneal nerve. The patient was diagnosed with postherpetic paresis with foot drop and carbamazepine $(400 \mathrm{mg} /$ day, oral) was initiated for the treatment of neuropathic pain. In addition, the patient started a physical therapy program including transcutaneous electrical nerve stimulation (TENS), ultrasound, massage, stretching and foot strengthening exercises. An ankle-foot orthotic was also prescribed and used to inhibit pathological motion. Three months after the herpes zoster diagnosis, a control EMG revealed some healing in the muscles innervated by the peroneal nerve and the presence of regeneration was considered. There was partial improvement in muscle function, the muscular strength of the ankle dorsiflexor and extensor hallucis longus improved to $2 / 5$ (left/right). A written informed consent was obtained from the patient.

However, the patient's pain persisted and he had unexplained weight loss (11.3\% of his body weight in three months, BMI: 22.1). Oral carbamazepine and

Table 2. Needle electromyography

\begin{tabular}{lccc}
\hline Muscle & Fibs/PSWs & MUAP & Recruitment \\
\hline Left tibialis anterior & $++l++$ & Normal & Reduced \\
Left peroneus longus & $++l++$ & Normal & Reduced \\
Left biceps femoris (short head) & None & Normal & Complete \\
Left gastrocnemius (medial head) & None & Normal & Complete \\
Left tensor fascia lata & None & Normal & Complete \\
Right tibialis anterior & None & Normal & Complete \\
Right peroneus longus & None & Normal & Complete \\
Right biceps femoris (short head) & None & Normal & Complete \\
Right gastrocnemius (medial head) & None & Normal & Complete \\
\hline
\end{tabular}

Fibs: Fibrillation potentials; PSWs: Positive sharp waves; MUAP: Motor unit action potential. 
physical therapy were discontinued. Laboratory test results included fasting blood glucose $317 \mathrm{mg} / \mathrm{dL}$, HbAlc 14.4\%; glucose (1000 $\mathrm{mg} / \mathrm{dL})$ and ketones $(5 \mathrm{mg} / \mathrm{dL})$ in urine. Clinical and laboratory findings revealed that the patient had previously undiagnosed diabetes mellitus. Insulin treatment was started with $\mathrm{NPH}$ and detemir. Three weeks after initiation of insulin therapy, the patient became normoglycemic, his pain was also reduced. The patient's ability to walk improved and foot drop disappeared almost completely. Antidiabetic pharmacotherapy was continued in an outpatient clinic. Two years after herpes zoster diagnosis, a follow-up evaluation showed that the muscular strength of the tibialis anterior improved to 5/5 (left/right) and the muscular strength of the peroneus longus and the extensor hallucis longuis muscles improved to $4 / 5$ (left/right). The EMG repeated at the final visit demonstrated significant healing had occurred and reduced numbers of fibrillation potentials and positive sharp waves.

\section{DISCUSSION}

Motor involvement in herpes zoster is very rare..$^{[1,8]}$ Most cases in the literature presented with ocular and facial paralysis. Segmental motor paralysis occurs in 0.5 to $5 \%$ of herpes zoster patients. ${ }^{[9]}$ On the other hand, neuropathic pain is a common diagnosis of herpes zoster infections. It may happen after (postherpetic) or before (preherpetic) the infection. Cvjetković et al. ${ }^{[10]}$ reported that people older than 60 years of age are the most common age group among herpes zoster patients suffering from preherpetic neuralgia. Herpes zoster and postherpetic neuralgia were associated with age, female sex, diabetes mellitus, cancer history, and HIV treatment. ${ }^{[1]}$ Some patients may have pain without developing the characteristic lesions, so diagnosis can be difficult. Therefore, all elderly and immunocompromised patients who present with neuropathic pain should be evaluated for the disease.

Foot drop characterized by weakness of ankle dorsiflexion is the common presentation of peroneal neuropathy. Clinical examination can assist with localizing the lesion. In a pure common peroneal neuropathy, there is weakness in ankle dorsiflexion, toe extension and foot eversion; however, the ankle plantar flexion and foot inversion are spared and the knee flexion is normal. Weakness during foot inversion is suggestive of sciatic neuropathy or lumbosacral plexopathy. Additionally, weak hip abduction indicates L5 radiculopathy, weak knee flexion indicates sciatic neuropathy. Electrodiagnosis is also useful in the evaluation of foot drop. In the early periods of an axonal degeneration, motor unit axonal potential (MUAP) morphology remains normal. In acute neuropathic lesions on needle EMG, MUAP changes take time, usually many weeks to months. Electromyography findings in our patient confirmed the diagnosis of partial axonal degeneration of left peroneal nerve, and there was a pattern of reduced recruitment of MUAPs with normal morphologies. Because no abnormalities were found in the hamstrings or distal tibial-innervated muscles, paraspinal EMG was not performed. Abnormalities in these muscles imply that the lesion is at the level of the sciatic nerve or higher. ${ }^{[12]}$

Treatment depends on the specific cause of the foot drop and includes orthotics, physical therapy, pharmacological and surgical treatment. Physical therapy plays an important role in the management of foot drop. Ankle-foot orthotic is the most common treatment, and may provide dorsiflexion assistance. Stretching and strengthening foot exercises help to improve gait and strengthen muscles. In some patients with foot drop, electrical nerve stimulation helps generate electrical impulses within the muscles and increase tone and contractility. Because of the silent progress of "undiagnosed diabetes", our patient did not respond well to physical treatment. Foot drop treatments may be directed at symptoms, but determining the underlying cause is usually effective to control and treat it.

This diabetic patient was diagnosed late due to the severity of the other neurological symptoms (pain, muscle weakness). Although he had typical signs of diabetes like polyuria and polydipsia, he did not complain about it. Nevertheless, his sudden weight loss indicated a systemic disease. Routine laboratory analyses should be done and checked at the initial visit. Diabetes can be diagnosed with laboratory findings. Moreover, it should be kept in mind during the differential diagnosis for peripheral neuropathy. Diabetic neuropathic cachexia is an uncommon presentation of diabetic neuropathy characterized by dramatic weight loss and severe neuropathic pain. ${ }^{[13]}$ The goal of treatment is to lower the high blood glucose levels in these cases. Insulin treatment leads to a rapid recovery from painful symptoms.

Diabetes increases the risk of developing herpes zoster in patients, particularly among elderly patients. ${ }^{[14,15]}$ Weitzman et al. ${ }^{[1]}$ reported that age, diabetes and cancer history were some of the factors associated with herpes zoster and postherpetic 
neuralgia. On the other hand, the literature review revealed only limited cases with postherpetic motor involvement. ${ }^{[16-18]}$ Although some cases had a history of diabetes or cancer, a relationship between these features and postherpetic motor involvement was not discussed in this article.

In conclusion, this report presents a possible association between postherpetic motor involvement and diabetes mellitus or another underlying systemic disease, particularly in elderly patients. If herpes zoster infection progresses to motor involvement, it is important to ask whether this unusual complication results from diabetes or another cause of immunodeficiency.

\section{Acknowledgment}

We would like to thank Prof. Dr. Lokman Öztürk, Ege University Department of Anatomy, for his comments and suggestions, which helped us to considerably improve the manuscript.

\section{Declaration of conflicting interests}

The authors declared no conflicts of interest with respect to the authorship and/or publication of this article.

\section{Funding}

The authors received no financial support for the research and/or authorship of this article.

\section{REFERENCES}

1. Kang SH, Song HK, Jang Y. Zoster-associated segmental paresis in a patient with cervical spinal stenosis. J Int Med Res 2013;41:907-13.

2. Niv D, Maltsman-Tseikhin A, Lang E. Postherpetic neuralgia: what do we know and where are we heading? Pain Physician 2004;7:239-47.

3. Thomas SL, Hall AJ. What does epidemiology tell us about risk factors for herpes zoster? Lancet Infect Dis 2004;4:26-33.

4. Niv D, Maltsman-Tseikhin A. Postherpetic neuralgia: the never-ending challenge. Pain Pract 2005;5:327-40.

5. Baron R. Mechanisms of disease: neuropathic pain--a clinical perspective. Nat Clin Pract Neurol 2006;2:95-106.

6. Schilling ML. Pain management in older adults. Curr Psychiatry Rep 2003;5:55-61.

7. Niccoli T, Partridge L. Ageing as a risk factor for disease. Curr Biol 2012;22:741-52.

8. Haanpää M, Häkkinen V, Nurmikko T. Motor involvement in acute herpes zoster. Muscle Nerve 1997;20:1433-8.

9. Rosenfeld T, Price MA. Paralysis in herpes zoster. Aust N Z J Med 1985;15:712-6.

10. Cvjetković D, Jovanović J, Hrnjaković Cvjetković I, Aleksić Dordević M. Clinical characteristics and prognostic significance of preherpetic neuralgia. Med Pregl 2002;55:412-4. [Abstract]

11. Weitzman D, Shavit O, Stein M, Cohen R, Chodick G, Shalev V. A population based study of the epidemiology of Herpes Zoster and its complications. J Infect 2013;67:463-9.

12. Masakado Y, Kawakami M, Suzuki K, Abe L, Ota T, Kimura A. Clinical neurophysiology in the diagnosis of peroneal nerve palsy. Keio J Med 2008;57:84-9.

13. Ellenberg M. Diabetic neuropathic cachexia. Diabetes 1974;23:418-23.

14. Guignard AP, Greenberg M, Lu C, Rosillon D, Vannappagari V. Risk of herpes zoster among diabetics: a matched cohort study in a US insurance claim database before introduction of vaccination, 1997-2006. Infection 2014;42:729-35.

15. Hata A, Kuniyoshi M, Ohkusa Y. Risk of Herpes zoster in patients with underlying diseases: a retrospective hospitalbased cohort study. Infection 2011;39:537-44.

16. Gunduz A, Yavuz D, Uzun N. Zoster paralysis secondary to different parts of peripheral nervous system. Archives of Neuropsychiatry 2012;49:314-6.

17. Takahama $H$, Tsukahara $N$, Hirayama $M$, Ito $S$, Sakuramoto C. Isolated double herpes zoster paresis involving the left facial nerve and the right peroneal nerve following disseminated herpes zoster. J Dermatol 2007;34:349-52.

18. Seo DH, Lee SJ, Hyun JK, Kim TU. A case of herpes zoster peripheral polyneuropathy manifested by foot drop in chronic myeloid leukemia. Ann Rehabil Med 2012;36:724-8. 